\title{
Long-term Visual and Anatomical Outcome after Surgical Management of Epiretinal Membrane
}

\author{
Lubna Feroz, Syed Fawad Rizvi, Syed Asaad Mahmood, Syeda Aisha Bokhari and Uzma Naz \\ Department of Ophthalmology, Layton Rahmatulla Benevolent Trust Tertiary Care Eye Hospital, Korangi, Karachi, Pakistan
}

\begin{abstract}
Objective: To assess the functional and surgical long-term outcomes following epiretinal membrane surgery.
Study Design: Case series.

Place and Duration of Study: Layton Rahmatulla Benevolent Trust Tertiary Care Eye Hospital, Karachi, from January 2016 to December 2020.

Methodology: A medical record review was carried out of patients, who had undergone surgical management of ERM and had presented for follow-up for at least three years. Best corrected visual acuity (BCVA) and Optical Coherence Tomography (OCT) parameter [(integrity of ellipsoid zone (EZ)] was evaluated. BCVA, using Snellen's chart, is performed routinely on each visit, which was converted to logMAR chart for analysis in this study. OCT was also performed to evaluate the integrity of outer retinal layers using Heidelberg OCT.

Results: Sixty-five eyes of 54 patients were included in the study, including 41 eyes of 36 men (63\%) and 24 eyes of 18 women (37\%). Mean age was $46.2 \pm 8.9$ years. The mean BCVA significantly improved from $0.88 \pm 0.28$ logMAR (6/45 Snellen) preoperatively to $0.64 \pm 0.21(6 / 27)$ at the end of first year $(p<0.001)$, which improved to $0.54 \pm 0.19(6 / 21)$ at the end of second year, and $0.53 \pm 0.20(6 / 20)$ after three years of follow-up. The post-op vision at three years was stratified according to the integrity of EZ on OCT performed at the same follow-up; and a significant difference was observed. EZ was intact in 52 eyes with a mean BCVA of $0.49 \pm 0.16 \log$ MAR (6/18 Snellen); while it was found disrupted in 13 eyes, where the BCVA was $0.68 \pm$ $0.26(6 / 29)$.
\end{abstract}

Conclusion: Anatomically intact outer retinal layer significantly correlated with improved BCVA.

Key Words: Epiretinal membrane, Optical coherence tomograghy, Best corrected visual acuity.

How to cite this article: Feroz L, Rizvi SF, Mahmood SA, Bokhari SA, Naz U. Long-term Visual and Anatomical Outcome after Surgical Management of Epiretinal Membrane. J Coll Physicians Surg Pak 2022; 32(02):226-229.

\section{INTRODUCTION}

Epiretinal membrane is a common vitreoretinal disease, which may result in visual disturbance. ${ }^{1}$ There is a contractile fibro-cellular proliferation at the surface of the sensory retina, which leads to deformation of its normal anatomical structure, leading to distortion of central vision. ${ }^{2,3}$ Epiretinal membrane can be idiopathic. Inflammation, retinal vascular diseases, trauma orsurgery are common causes of secondary ERM. ${ }^{4}$ Secondary ERMs are found to be more visual disturbing than idiopathic. As metamorphopsia is the most common symptom in ERM, patients frequently present with complaints of distortion of vision resulting in difficulty in their daily life activities, that causes social distress. ${ }^{5}$ However, decrease in visual acuity might also occur secondary to ERM. Hence, when such membranes cause marked distress in everyday activities, there arise the need to treat those membranes.

Correspondence to: Dr. Lubna Feroz, Department of Ophthalmology, Layton Rahmatulla Benevolent Trust Tertiary Care Eye Hospital, Korangi, Karachi, Pakistan E-mail: lubna_mallick@live.com

Received: February 05, 2021; Revised: May 31, 2021;

Accepted: June 16, 2021

DOI: https://doi.org/10.29271/jcpsp.2022.02.226
Removal of the ERM is a well-established surgical procedure, which is indicated if the patient is symptomatic or if their vision has been adversely affected. ${ }^{6}$ Pars plana vitrectomy (PPV) and peeling of membrane, is the established surgical treatment for ERM. ${ }^{7}$ Additional removal of the internal limiting membrane (ILM) has been reported to decrease the chance of recurrence of ERM, which act as a scaffold for ERM, after surgery. It is considered a safe procedure and also provides favourable anatomical outcome. However, despite the successful removal of ERM, sometimes the physiological outcomes remain unsatisfactory. ${ }^{8}$

Optical coherence tomography (OCT) is a non-invasive method of imaging, where spectral-domain OCT (SD-OCT) represents the standard for in vivo imaging in both research and clinical applications. Introduction of OCT has significantly improved the interpretation of macular diseases and their outcomes. ${ }^{9,10}$ However, conventional time-domain (TD) OCT with approximately 10 microns axial resolution is limited in its ability to show detailed pathologic changes, especially of the photoreceptor layer. The introduction of high-resolution OCT systems improved the visualisation of these intraretinal layers and of the vitreomacular interface.$^{11}$ Among other pathologies of macula, OCT is known to provide significant knowledge regarding anatomical changes that occur in epiretinal membrane, both 
pre-operatively and post-operatively. Therefore, the evaluation of layers of macula and its integrity has greater significance in predicting the functional and surgical outcomes of ERM, ${ }^{12}$ where one can correlate the results of surgery with that of the OCT reports of the patient easily.

The objective of the present study was to determine the longterm outcomes following the ERM surgery including evaluation of outer retinal layer integrity as well as improvement in BCVA.

\section{METHODOLOGY}

This retrospective case series was carried out at Layton Rahmatulla Benevolent Trust Tertiary Care Eye Hospital, Karachi. Patient enrollment was done by using non-probability consecutive sampling technique. Approval was obtained from the Hospital Ethics Board before the commencement of the study.

Medical charts and OCT records of patients of both genders, who had undergone surgical management for symptomatic ERM from January 2016 to December 2017 and had maintained follow-up for at least three years, were included in the study. Data was collected and analysed in December 2018, 2019, and finally in December 2020.

Excluded were patients who had other associated eye diseases, which could impact the patient's visual status, such as corneal opacities, patients with significant cataract (nuclear sclerosis grade +3 and more, posterior subcapsular cataract grade +2 or more), advanced glaucoma, history of retinal detachments, history of amblyopia, etc. Only those patients who had symptomatic ERM and those with nuclear sclerosis grade +1 and pseudophakia, were included in the study.

As per hospital protocol, preoperative OCT (Spectralis, Heidelberg Engineering) is regularly performed for cases of ERM and then performed as and when thought to be necessary by the retinal surgeon on follow-up visits.

Best corrected visual acuity using Snellen's chart is performed routinely on each visit, which was converted to logMAR chartfor analysis in this study. Complete slit-lamp examination, intraocular pressure assessment with applanation tonometer; and fundoscopy was done with 78D (Volk double aspheric lens) for the examination of posterior pole; and peripheral retina was examined with Goldmann 3 mirror lens.

Spectral domain optical coherence tomography (Spectralis, Heidelberg Engineering) was also performed to evaluate the integrity of outer retinal layers. Informed consent was taken from patients, who fulfilled the criteria for the study, after the explanation of merits and demerits of the surgery.

The surgical procedure in all cases was a 3-port 25-gauge pars plana vitrectomy (PPV). After taking all aseptic measures, it was performed under local peri-bulbar anesthesia [with a mixture of lidocaine (2\%) and bupivacaine $(0.7 \%), 3-4 \mathrm{ml}$. Small guage vitrectomy was performed on constellation vision system (Alcon Surgical) by a single surgeon. After core vitrectomy and removal of posterior hyaloid (where needed), staining was done with $0.5 \mathrm{ml}$ of $0.05 \%$ of indocyanine green (ICG) dye, and peeling of the ERM followed by peeling of ILM, which was performed with the help of end-grasping vitreo-retinal forceps. Any complication during the surgery or postoperatively was addressed and managed, accordingly.

Patients were then called and examined on the first day of postoperation then at first week. Thereafter they had been called at $1^{\text {st }}$ month then at $1^{\text {st }}, 2^{\text {nd }}$ and finally at $3^{\text {rd }}$ year of their surgery. On each visit, the patients, were assessed for their best corrected visual acuity; both anterior and posterior examination was performed, and finally retinal layers are being examined using SD-OCT.

Data was analysed using IBM SPSS version 25 , and paired t-test was used for comparison of visual status. Frequencies with percentages were used to present qualitative variables and mean \pm SD were calculated for the quantitative variables. The postoperative vision at three years was stratified, according to the integrity of EZ on OCT performed. Gain in visual acuity by two lines is considered to be statistically significant, using logMar chart. Independent sample t-test was applied to determine the significance of improvement in BCVA. P-value $\leq 0.05$ was taken as significant.

\section{RESULTS}

Records of 65 eyes in 54 patients were identified for symptomatic epiretinal membrane surgery during this period; and had maintained follow-up postoperatively for three years. The decision to proceed for surgery in all cases was either BCVA $<6 / 18$ or presence of metamorphopsia.

There were 41 eyes of 36 men (63\%) and 24 eyes of 18 women (37\%). Mean age was $46.2 \pm 8.9$ years. The mean BCVA improved from $0.88 \pm 0.28$ logMAR (6/45 Snellen) preoperatively to $0.64 \pm 0.21(6 / 27)$ at the end of first year, which was statistically significant $(p<0.001)$. This further improved to $0.54 \pm 0.19(6 / 21)$ at the end of second year; and $0.53 \pm 0.20$ (6/20) after three years of follow-up. When comparing BCVA using paired sample t-test, the difference was statistically significant at one year to two years $(p<0.001)$, but the improvement between twoyears to three years of follow-up was non-significant $(p=0.174)$.

The postoperative vision at three years was stratified according to the integrity of EZ on OCT performed at the same follow-up; and a significant difference was observed. EZ was intact in 52 eyes with a mean BCVA of $0.49 \pm 0.16$ logMAR (6/18 Snellen); while it was found disrupted in 13 eyes, where the BCVA was $0.68 \pm 0.26(6 / 29)(p=0.020)$.

\section{DISCUSSION}

Epiretinal membrane is a fibrocellular membrane, which causes disruption in the vitreomacular interface anatomy. Its sheen like reflex sheet, which is translucent, and have irregular borders and surfaces, found on the inner surface of the ILM at the macula. The chief complaint is the distortion of vision and the images appear either small or distorted. However, blurring 
of vision and even severe visual loss can also occur. Its prevelance is $7.6 \%$ in a general population with no gender predilection. ${ }^{13}$ Epiretinal membranes are generally treated only when they become symptomatic and causing disruption in the macular anatomy. The standard treatment for the ERM is vitrectomy with the removal of membrane with or without ILM removal. If these membranes are left untreated, the significant image distortion might affect the patient's quality of life. Though, many ERMs are asymptomatic and non-progressive, population studies have shown the rate of progression of ERMs to be between 16 and 33\%. It has been reported that ERM might regress in one-fourth of the cases. It is, therefore, important to identify when to intervene with surgery. ${ }^{14}$ Visual acuity is commonly used as an indicator for timing of surgical intervention and its outcomes. Here, only those patients were included, who were significantly symptomatic, that is their quality of life has been affected adversely.

This study included the patients who underwent ERM surgery along with ILM peeling, in which both idiopathic and secondary ERMs were included. The patients were followed for more than two years, which falls among the longer follow-ups reported in the literature. ${ }^{15}$ The main finding was, BCVA continued to improve up to two years after surgery, which was statistically significant and remained stable for one year after that. The other significant finding was that the integrity of ellipsoid zone $(E Z)$, which is one of the outer retinal layers, was found to be associated with improved BCVA. This shows that the intact ellipsoidzone seen onSD-OCT is one of the importantfactorsin determining the post-op BCVA, which is also supported by Jeon et al. and others. ${ }^{16-18}$ On the other hand, patients with disrupted ellipsoid zone there was poor visual recovery as compared to others. Therefore, SD-OCT has got remarkable importance in determining the anatomical as well as the physiological outcomes for ERM surgery, ${ }^{19}$ where the present authorscan assess postoperatively the anatomical outcome by observing the status of outer retinal layers and also can correlate it with the visual status of the patient.

Previous studies have supported that post-surgically, the decrease in macular thickness improves postoperative visual acuity. However, they did not establish any correlation between the individual layer and its integrity. ${ }^{20}$ In this study, the individual outer retinal layer was actually observed and then correlated with the visual acuity.

It was also observed that even if the BCVA does not improve after surgery, there may be an improvement in the metamorphopsia, which itself is a significant visual symptom marker. ${ }^{21}$ As many patients do not have decreased visual acuity but observe image distortion; hence, its improvement may bring some satisfaction to the patients and improve their daily life acitvities.

This study also showed that those patients who had ERM secondary to coexisting ocular pathologies, such as diabetic retinopathy or central retinal vein occlusion, presented with poorer visual outcomes, while those with idiopathic ERM showed better visual results. It can be explained by the fact that patients with other ocular pathologies already have compromised and ischemic retina, which may be a main cause of poor vision. $^{22,23}$

The limitations of the present study are its retrospective nature. Secondly, the authors did not evaluate other layers of retina and their correlation with the visual acuity. Furthermore, due to retrospective study and long duration, the preoperative OCT data of few of the patients could not be recovered. Therefore, status of retinal layers before surgery could not be assessed, which might have altered the surgical outcomes. Nonetheless, this study shows the significance of postoperative intact ellipsoid zone as the better anatomical as well as visual outcome; and this study is one of those who had longer follow-up to predict the changes that may occur lately afterERM surgery.

\section{CONCLUSION}

Improvements in visual acuity, after surgical management of ERM, can be appreciated even at two years of follow-up. Normal integrity of ellipsoid zone has significant association with improved BCVA.

\section{ETHICALAPPROVAL:}

Approval was obtained from the Hospital Ethics Board before the commencement of the study.

\section{PATIENTS' CONSENT:}

Informed consents were taken from patients, who fulfilled the criteria for the study, after the explanation of merits and demerits of the surgery

\section{CONFLICT OF INTEREST:}

The authors declared no conflict of interest.

\section{AUTHORS' CONTRIBUTION:}

LF: Study design, literature search.

SFR: Literature review, initial drafting.

SAM: Statistical analysis, data analysis.

SAB: Manuscript editing and review.

UN: Critical review ofmanuscript forfinal approval.

\section{REFERENCES}

1. Folk JC, Adelman RA, Flaxel CJ. Idiopathic epiretinal membraneand vitreomacular traction preferred practice pattern (R) guidelines. Ophthalmol 2016; 123(1):152-81. doi:10.1016/j.ophtha.2015.10.048.

2. Matsumoto C, Arimura E, Okuyama S, Takada S, Hashimoto S, Shimomura Y. Quantification of metamorphopsia in patients with epiretinal membranes. Invest Ophthalmol Vis Sci 2003; 44(9):4012-6. doi: 10.1167/iovs.03-0117.

3. Takagi S, Kudo S, Yokota H, Akiba M, Mandai M, Hirami Y, et al. Assessment of the deformation of the outer nuclear layer in the Epiretinal membrane using spectral-domain optical coherence tomography. BMC Ophthalmol 2019; 19(1):113. doi.org/10.1186/s12886-019-1124-z.

4. Bu SC, Kuijer R, Li XR, Hooymans JM, Los LI. Idiopathic epiretinal membrane. Retina 2014; 34(12):2317-35. doi: 


\subsection{7/IAE.0000000000000349.}

5. Ichikawa $Y$, Imamura $Y$, Ishida M. Metamorphopsia and tangential retinal displacement after epiretinal membrane surgery. Retina 2017; 37(4):673-9. doi: 10.1097/IAE. 0000000000001232.

6. Massin P, Allouch C, Haouchine B, Metge F, Paques M, Tangui $L$, et al. Optical coherence tomography of idiopathic macular epiretinal membranes before and after surgery. Am J Ophthalmol 2000; 130(6):732-9. doi: 10.1016/s00029394(00)00574-2.

7. González-Saldivar G, Berger A, Wong D, Juncal V, Chow DR. Ectopic inner foveal layer classification scheme predicts visual outcomes after epiretinal membrane surgery. Retina 2020; 40(4):710-7. doi: 10.1097/IAE.0000000000002486.

8. Shimozono M, Oishi A, Hata M, Matsuki T, Ito S, Ishida K, et al. The significance of cone outer segment tips as a prognostic factor in epiretinal membrane surgery. Am J Ophthalmol 2012; 153(4):698-704.e1. doi:10.1016/j. ajo.2011.09.011.

9. Gattoussi S, Buitendijk GH, Peto T, Leung I, SchmitzValckenberg S, Oishi A, et al. The European Eye Epidemiology spectral-domain optical coherence tomography classification of macular diseases for epidemiological studies. Acta Ophthalmol 2019; 97(4):364-71. http://doi.org/10.1111/ aos.13883.

10. Bhende M, Shetty S, Parthasarathy MK, Ramya S. Optical coherence tomography: A guide to interpretation of common macular diseases. Indian J Ophthalmol 2018; 66(1):20. http://doi.org/10.4103\%2Fijo.IJO_902_17.

11. Falkner-Radler Cl, Glittenberg C, Hagen S, Benesch T, Binder $S$. Spectral-domain optical coherence tomography for 2onitoring epiretinal membrane surgery. Ophthalmol 2010; 117(4):798-805. doi:10.1016/j. ophtha.2009.08.034.

12. Elhusseiny AM, Flynn Jr HW, Smiddy WE. Long-term outcomes after idiopathic epiretinal membrane surgery. Clin Ophthalmol 2020; 14:995-1002. http://doi.org/10. 2147\%2FOPTH.S242681.

13. Koh V, Cheung CY, Wong WL, Cheung CM, Wang JJ, Mitchell $P$, et al. Prevalence and risk factors of epiretinal membrane in Asian Indians. Invest Ophthalmol Vis Sci 2012; 53(2):1018-22. doi: 10.1167/iovs.11-8557.

14. Dawson S, Shunmugam M, Williamson T. Visual acuity outcomes following surgery for idiopathic epiretinal membrane: An analysis of data from 2001 to 2011. Eye 2014; 28:219-24. doi.org/10.1038/eye.2013.253.

15. Kim J, Rhee KM, Woo SJ, Yu YS, Chung H, Park KH. Longterm temporal changes of macular thickness and visual outcome after vitrectomy for idiopathic epiretinal membrane. Am J Ophthalmol 2010; 150(5):701-9. doi: 10.1016/j.ajo.2010.05.037.

16. Jeon S, Jung B, Lee WK. Long-term prognostic factors for visual improvement after epiretinal membrane removal. Retina 2019; 39(9):1786-93. doi: 10.1097/IAE.000000 0000002211.

17. Houly JR, Veloso CE, Passos E, Nehemy MB. Quantitative analysis of external limiting membrane, ellipsoid zone and interdigitation zone defects in patients with macular holes. Graefe's Arch Clin Exp Ophthalmol 2017; 255(7):1297-306. doi: 10.1007/s00417-017-3636-7.

18. Iuliano L, Fogliato G, Gorgoni F, Corbelli E, Bandello F, Codenotti M. Idiopathic epiretinal membrane surgery: Safety, efficacy and patient related outcomes. Clin Ophthalmol 2019; 13:1253-65. doi: 10.2147/OPTH.S17 6120.

19. Sheales MP, Kingston ZS, Essex RW. Associations between preoperative OCT parameters and visual outcome 3 months postoperatively in patients undergoing vitrectomy for idiopathic epiretinal membrane. Graefe's Arch Clin Exp Ophthalmol 2016; 254(10):1909-17. doi: 10.1007/s00 417-016-3326-x.

20. Hecht I, Yeshurun I, Bartov E, Bar A, Burgansky-Eliash Z, Achiron A. Retinal layers thickness changes following epiretinal membrane surgery. Eye 2018; 32(3):555-62. doi: 10.1038/eye.2017.233.

21. Klein R, Klein BE, Wang Q, Moss SE. The epidemiology of epiretinal membranes. Trans Am Ophthalmol Soc 1994; 92:403-25.

22. Yazici AT, Alagöz N, Celik HU, Bozkurt E, Alagöz C, Cakir M, et al. Idiopathic and secondary epiretinal membranes: Do they differ in terms of morphology? An optical coherence tomography-based study. Retina 2011; 31(4):779-84. doi: 10.1097/IAE.0b013e3181ef8786.

23. Stevenson W, Ponce CM, Agarwal DR, Gelman R, Christoforidis JB. Epiretinal membrane: Optical coherence tomography-based diagnosis and classification. Clin Ophthalmol 2016; 10:527-34. doi: 10.2147/OPTH.S97722. 